\title{
Modeling of the Total Electronic Content and magnetic field anomalies generated by the 2011 Tohoku-oki tsunami and associated acoustic-gravity waves
}

\author{
E. Alam Kherani, Philippe Lognonne, H. Herbert, L. Rolland and Eurico R.de Paula \\ INPE SJC, Brazil/IPGP, France/GeoAzur, France
}

Copyright 2013, SBGf - Sociedade Brasileira de Geofísica

This paper was prepared for presentation at the $13^{\text {th }}$ International Congress of the Brazilian Geophysical Society, held in Salvador, Brazil, August 24-28 2013.

Contents of this paper were reviewed by the Technical Committee of the $13^{\text {th }}$ International Congress of The Brazilian Geophysical Society and do not necessarily represent any position of the SBGf, its officers or members. Electronic reproduction represent any position of the SBGf, its officers or members. Electronic reproduction
or storage of any part of this paper for commercial purposes without the written consent of The Brazilian Geophysical Society is prohibited.

\section{Abstract}

In the present work, numerical simulations of the atmospheric and ionospheric anomalies are performed for the Tohoku-Oki tsunami (11 March 2011). The Tsunami-Atmosphere-Ionosphere (TAI) coupling mechanism via acoustic gravity waves (AGWs) is explored theoretically using the TAI coupled model. For the modeled tsunami wave as an input, the coupled model simulates the wind, density and temperature disturbances or anomalies in the atmosphere and electron density/magnetic anomalies in the $F$ region of the ionosphere. Also presented are the GPS-TEC and ground-based magnetometer measurements during first hour of tsunami and good agreements are found between modeled and observed anomalies. At first, within 6 minutes from the tsunami origin, the simulated wind anomaly at $250 \mathrm{~km}$ altitude and TEC anomaly appear as the dipole-shaped disturbances around epicenter, then as the concentric circular wavefronts radially moving away from the epicenter with the horizontal velocity $\sim 800 \mathrm{~m} / \mathrm{s}$ after 12 minutes followed by the slow moving (horizontal velocity $\sim 250 \mathrm{~m} / \mathrm{s}$ ) wave disturbance after $\mathbf{3 0}$ minutes. The detailed verticalhorizontal propagation characteristics suggest that the anomalies appear before and after 30 minutes are associated with the acoustic and gravity waves respectively. Similar propagation characteristics are found from the GPS-TEC and magnetic measurements presented here and also reported from recent studies. The modeled magnetic anomaly in the $F$ region ionosphere is found to have similar temporal variations with respect to the epicenter distance as that of the magnetic anomaly registered from the ground-based magnetometers. The high frequency component $\sim 10$ minutes of the simulated wind, TEC and magnetic anomalies in the $F$ region develops within 6-7 minutes after the initiation of the tsunami, suggesting the importance of monitoring the highfrequency atmospheric/ionospheric anomalies for the early warning. These anomalies are found to maximize across the epicenter in the direction opposite to the tsunami propagation suggesting that the large atmospheric/ionospheric disturbances are excited in the region where tsunami does not travel.

Introduction

The magnitude 9.0 Tohoku-Oki earthquake occurred at 05:46:23 UT off the east coast of Honshu, Japan (38.322N, 142.369E). Waves generated in the solid earth, ocean, atmosphere and ionosphere were detected by acceleration, displacement and pressure sensors on the Earth surface and ocean bottom, [e.g. Nishimura et al., 2011, Maeda et al., 2011] and also by various ionospheric sensors such as GPS dual frequency sensor and allsky imagers monitoring the anomalies in the ionosphere [Makela et al, 2011; Heki et al, 2011; Maruyama et al, 2011]. Following the pioneering observations of Calais and Minster [1995] and Artru et al [2005] for seismic and tsunamigenic signals, these ionospheric anomalies are interpreted on the basis of the dynamic coupling of the solid/ocean with the atmospheric and ionospheric systems Lognonne et al, [1998] for the interior/atmospheric (See Lognonne seismic coupling, Artru et al [2005], Occhipinti et al, [2008] for the tsunami waves, Kherani et al, [2009] for the acoustic-ionospheric waves coupling). According to this coupling mechanism, the surface/sea level vertical displacement during earthquake leads to the excitation of Acoustic Gravity wave in the atmosphere. These waves excite the wind disturbances in the atmosphere (referred as Co-seismic Atmospheric Disturbances or CADs) and TEC disturbances in the ionosphere (referred as Co-seismic lonospheric Disturbances or CIDs).

Tsunami-Atmosphere-Ionosphere Coupled Simulation model

The TAI coupling mechanism is accomplished in three steps: (i) the excitation of tsunami wave during the Tohoku-Oki tsunami, (ii) the excitation of Acoustic-Gravity Waves (AGWs) by the tsunami wave and (iii) the excitation of ionospheric anomalies by AGWs. The tsunami wave modeling is performed following the method described by Hebert et al [2006], by using the preliminary source of USGS for Tohoku-Oki tsunami (provided at http://earthquake.usgs.gov/earthquakes) and is used as a input in the present model. The 2nd and 3rd steps of the coupling is accomplished using the nonlinear 3D computational modeling [Kherani et al, 2009]. Kherani et al [2011] have derived the wave equation of AGWs using Navier-Stokes equations namely the continuity, momentum and energy equations in the atmosphere. We start from the Navier-Stokes equation and assume that the space variations of the viscosity are small compared to the 
wavelength of the gravity waves, which lead to

$$
\begin{gathered}
\frac{\partial \vec{W}^{\prime}}{\partial t}+\vec{W}^{\prime} \cdot \nabla \vec{W}^{\prime}=-\frac{1}{\rho} \nabla p+\vec{g}+\frac{1}{\rho}\left(\mu \nabla^{2} \vec{W}^{\prime}+\left(\zeta+\frac{\mu}{3}\right) \nabla\left(\nabla \cdot \vec{W}^{\prime}\right)\right) \\
\frac{\partial \rho}{\partial t}+\nabla \cdot\left(\rho \vec{W}^{\prime}\right)=0 \\
\frac{\partial p}{\partial t}+\left(\vec{W}^{\prime} \cdot \nabla\right) p+\gamma p \nabla \cdot \vec{W}^{\prime}=0
\end{gathered}
$$

where $\vec{W}^{\prime}=\vec{W}+\vec{W}_{o}, \vec{W}$ is the perturbation wind, $W_{o}$ is the background wind derived from the HWM model, $p=\frac{R \rho T}{\mathscr{M}_{m}}$ is the pressure, $(\rho, \mathrm{T})$ are the atmospheric mass density and temperature, while $\mu$ and $\zeta$ is the dynamic first and second viscosity and $R$ and $\mathscr{M}_{m}$ are the perfect gas constant and molar mass respectively. No assumption of linearity is made in writing $\vec{W}^{\prime}=\vec{W}+\vec{W}_{o}$ and the perturbation wind $\vec{W}$ may become of the same order as the mean background wind $\vec{W}_{o}$ i.e. above equations keep non-linearity in $\vec{W}$. By taking the time derivative of the momentum equation for the wind $\vec{W}^{\prime}$, and again substituting time derivatives of the density $(\rho)$, wind $\left(\vec{W}^{\prime}\right)$ and pressure $(p)$ from the NavierStokes equations, the wave equation for the wind $\vec{W}^{\prime}$ of AGWs is obtained in the following form:

$$
\begin{array}{r}
\frac{\partial^{2} \vec{W}^{\prime}}{\partial t^{2}}=\frac{1}{\rho} \nabla\left(\gamma p \nabla \cdot \vec{W}^{\prime}\right)-\frac{\nabla p}{\rho} \nabla \cdot\left(\rho \vec{W}^{\prime}\right)+\frac{1}{\rho} \nabla\left(\vec{W}^{\prime} \cdot \nabla\right) p \\
+\frac{\partial}{\partial t}\left(\nu \nabla^{2} \vec{W}^{\prime}+\left(\zeta^{\prime}+\frac{v}{3}\right) \nabla\left(\nabla \cdot \vec{W}^{\prime}\right)\right) \\
-\frac{\partial}{\partial t}\left(\vec{W}^{\prime} \cdot \nabla \vec{W}^{\prime}\right)
\end{array}
$$

Here $v=\mu / \rho, \zeta^{\prime}=\zeta / \rho$ are the first and second kinematic viscosities.

The hydromagnetic equations in the ionosphere are written as follows [Kherani et al, 2009]:

$$
\begin{gathered}
\frac{\partial \vec{u}_{s}}{\partial t}=\frac{q_{s}}{m_{s}}\left(\vec{E}+\vec{u}_{s} \times \vec{B}_{o}\right)-v_{s} \vec{u}_{s}+v_{s} \vec{W}^{\prime}, \\
\frac{\partial n_{s}}{\partial t}+\nabla \cdot\left(n_{s} \vec{u}_{s}\right)=P-L \\
\nabla^{2} \vec{E}-\nabla(\nabla \cdot \vec{E})-\frac{1}{c^{2}} \frac{\partial^{2} \vec{E}}{\partial t^{2}}-\mu_{o} \frac{\partial \vec{J}}{\partial t}=0 \\
\vec{J}=\underline{\sigma} \cdot \vec{E}+\vec{J}_{w} ; \quad \vec{J}_{w}=e\left(n_{i} \vec{u}_{i}-n_{e} \vec{u}_{e}\right) \\
\nabla^{2} \vec{B}-\frac{1}{c^{2}} \frac{\partial^{2} \vec{B}}{\partial t^{2}}=-\mu_{o} \nabla \times \vec{J} .
\end{gathered}
$$

Here $\left(n_{s}, \vec{u}_{s}\right)$ are the number density, velocity of plasma fluid 's' (s=ions(i)/electrons(e)), $\left(q_{i, e}=+Z_{i} e,-e\right),(\vec{W})$ is the amplitudes of AGWs from (1), $v_{s}$ is the frequency of collision between species s-to neutral, $\vec{B}_{o}$ is the Earth's magnetic field and $\vec{J}_{w}$ is the ionospheric current density caused by the AGWs, $(\vec{E}, \vec{J}, \vec{B})$ in above equations are the fluctuating electric field, net current and magnetic field in the ionosphere, $\sigma$ is the ionospheric conductivity tensor and $\left(c=\frac{1}{\sqrt{\mu_{o} \varepsilon_{o}}}\right)$ is the speed of light in vacum. $P, L$ are the production and loss of ions and electrons by photo ionization and chemical reactions. In addition to the wave equation (7), the electric field, $\vec{E}$, also satisfies the charge neutrality condition given by following equation:

$$
\nabla \cdot \vec{J}=0 \text { or } \nabla \cdot\left(\sigma \cdot \vec{E}+\vec{J}_{w}\right)=0 \Rightarrow \nabla \cdot \vec{E}=-\sigma^{-1}\left(\vec{E} \cdot \nabla \sigma+\nabla \cdot J_{w}\right) \text {. }
$$

Equations (2-10) form the closed set of equations to study the temporal and spatial variation of AGWs wind $\vec{W}$, atmospheric density/pressure $(\rho, p)$, ionospheric number density $(n)$, electric field $\vec{E}$ and magnetic field $\vec{B}$ anomalies. The time $\mathrm{t}=0$ in the simulation corresponds to the origin time $(5: 46: 23 \mathrm{UT})$ of the tsunami. At $\mathrm{t}=0$, ambient atmosphere and ionosphere $\left(p_{o}, \rho_{o}, n_{o}, v, T\right)$ are obtained from SAMI2 model.

Equations (2-10) are solved numerically using finitedifference method in three dimension that is consists of altitude, latitude and longitude. The implicit CrankNicholson scheme is employed to perform the time integration leading to the matrix equation that is subsequently solved by the Successive-Over-Relaxation method. The magnetic dipole coordinate system $(p, q, \phi)$ is adopted where $p, q, \phi$ represent the coordinates outward normal to the Earth's magnetic field, northward directed parallel to the Earth's magnetic field and azimuth angle (+ve towards west) respectively. The N-S and E$\mathrm{W}$ boundaries of simulation volume are $23-50^{\circ} \mathrm{N}$ and $131-156^{\circ} \mathrm{E}$ which covers the region of interest. The lower boundary for the atmosphere and ionosphere are chosen to be the ocean surface and $160 \mathrm{~km}$ respectively. The upper boundary is chosen to be $600 \mathrm{~km}$ for both atmosphere and ionosphere. At the lower boundary i.e at the ocean surface-atmosphere interface, the outward normal component $W_{p}$ of the wind $\vec{W}$ is continuous and is equals to $W_{T}$ for all time where $W_{T}$ is the output of the tsunami model as shown in Figure 2 and is changing with time. The tsunami wave-field, $W_{T}$, is obtained using the tsunami model with the time resolution of 1 minutes [Herbert et al, 2006].

\section{Results and Discussion}

In Figure 1, the snap shots of the tsunami wave-field $W_{T}$ generated by the tsunami at few selected time (between 5:46:23UT-6:46:23 UT) are shown. The origin time of tsunami is the first frame in Figure 1 when simulated tsunami wave-field has first finite vertical displacement and it corresponds to 5:46:23UT and will be referred as the origin time here. In Figure 2, the vertical propagation of the simulated CADs (here referred to the $p$ component, $W_{p}$, of the AGW) is shown at few selected times (after the origin time) in the altitude-longitude plane at fixed latitude $\left(=38.32^{\circ}\right)$. To infer the horizontal propagating characteristics of the CADs/CIDs, the travel-time diagram (TTD) of the input tsunami wave-field, simulated CADs (at $250 \mathrm{~km}$ ) and CIDs are plotted as a function of the distance to the epicenter versus the time after the origin time in Figures $3 a-3 c$ respectively. These CADs and CIDs are obtained along the line that cuts through the horizontal plane as shown in the last panel in Figure 1. The CADs and CIDs in these figures are low-pass filtered (periods greater than 10 minutes are allowed to pass) of the simulated CADs/CIDs. For comparison, the travel-time diagram of the observed CIDs from the GPS are plotted for $\mathrm{PRN}=09$ in Figure 3d. In these plots, various lines with different slopes are drawn to identify the various horizontal velocities of the wavefronts of the CADs/CIDs and GPS-CID. For the synthetic CADs/CIDs in 3b-3c, clockwise upper and lower velocity limits are shown as solid lines and correspond to the $800 \mathrm{~m} / \mathrm{s}$ and $250 \mathrm{~m} / \mathrm{s}$ respectively. In addition, two intermediate velocities are also shown as dashed lines corresponding to the $600 \mathrm{~m} / \mathrm{s}$ and $300 \mathrm{~m} / \mathrm{s}$ respectively. For 
the GPS-CIDs, clockwise, four lines with different slopes are shown representing the velocities equals to $1 \mathrm{~km} / \mathrm{s}, 550$ $\mathrm{m} / \mathrm{s}, 270 \mathrm{~m} / \mathrm{s}$ and $170 \mathrm{~m} / \mathrm{s}$ respectively.

The tsunami wave field in Figure 1 reveals the successive appearance of two tsunami wavefronts: the tsunami initial wavefront (dark red/dark blue) in the vicinity of the epicenter at the beginning, that spreads away as time grows. Modeling results in Figure 2 reveal that the initial CADs induced by the tsunami in the bottom of the atmosphere propagates upward and spreads horizontally as time progress. The CADs in the form of the longer wavelength acoustic gravity wave arrives to the thermospheric height $(\sim 200 \mathrm{~km})$ within 6 minutes that is followed by the shorter wavelength AGWs after 24 minutes. These two waves are likely associated with two different propagation regimes. The first one is primarily the longwavelength AGWs (or acoustic wave), with the velocity $\sim 600 \mathrm{~m} / \mathrm{s}$ (i.e. traveling $200 \mathrm{~km}$ altitude in 6 minutes) which is within the acoustic speed range in the thermosphere (Figure 1b), while the second one is the short-wavelength AGWs (or gravity wave), with the smaller phase velocities $\sim 150 \mathrm{~m} / \mathrm{s}$ (i.e. traveling $200 \mathrm{~km}$ altitude in 24 minutes) typical of the gravity waves ( e.g. $250 \mathrm{~m} / \mathrm{s}$ or below). The comparison with Figure 2 suggests that the acoustic wave and gravity wave are caused by the primary wavefront associated with the rupture and the tsunami propagation respectively.

It may be noted from Figure 2 that the CADs associated with the initial impulse or uplift propagates vertical during first 6 minutes and then gradually begins to spread horizontally in the thermosphere. This is in accordance with the mechanism proposed by Heki and Ping [2005] explaining that the observed TEC anomaly during TokachiOki earthquake may have been excited by co-seismic uplifts of the sea surface initially as upward propagating compressive pulses of atmosphere, which were gradually refracted to propagate horizontally in the ionosphere. The TTD of the input tsunami wave-field in Figure $3 a$ reveals the presence of wavefront moving with tsunami velocity $200 \mathrm{~m} / \mathrm{s}$. From the TTD of the simulated CADs/CIDs as shown in figures $3 b-3 c$, the presence of the high-frequency ( $\sim 10$ minutes) acoustic wavefront near the epicenter before 30 minutes and low frequency (¿10 minutes) gravity wavefronts far away from the epicenter after 30 minutes are identified (10 minutes is the typical Brunt-vaisala period in the thermosphere). Moreover, with time, the slope of the wavefront i.e. the velocity of the CADs/CIDs gradually decreases from $800 \mathrm{~m} / \mathrm{s}$ to $250 \mathrm{~m} / \mathrm{s}$. The upper limit of $800 \mathrm{~m} / \mathrm{s}$ is determined by the the acoustic speed which is $\sim 800 \mathrm{~m} / \mathrm{s}$ at $250 \mathrm{~km}$ (Figure 1b) while lower limit of $250 \mathrm{~m} / \mathrm{s}$ is determined by the tsunami propagation. The TTD of the observed CIDs as shown in Figure 3d exhibits similar wave features as those reported recently by Rolland et al [2011]. Also, the transition from acoustic to gravity wavefront with increasing epicenter distance and time is noted in both observed and synthetic TTDs.

\section{Summary}

The major findings in this study are summarized as follows:

1. The simulated CADs/CIDs appear as the disk-shaped anomaly at the ionospheric height $(250 \mathrm{~km})$ within 6-7 minutes. This is followed (at 12 minutes from the origin time of tsunami) by the development of the concentric circular-shaped anomaly moving away from the CADs/CIDs origin. These are found to be associated with the high-frequency-long-wavelength AGWs (or acoustic wave) excited by the initial impulse or rupture. Later at 27 minutes, the new wavefronts, in the form of the elongated disk-shaped anomaly begins to appear in the vicinity of CADs/CIDs origin. These new wavefronts are found to be associated with the low-frequency-short-wavelength AGWs (or gravity / tsunami wave) with vertical phase velocity $<200 \mathrm{~m} / \mathrm{s}$ and horizontal phase velocity $\sim 250 \mathrm{~m} / \mathrm{s}$.

2. CADs, CIDs and magnetic anomalies are found to maximize in the forward direction of tsunami propagation across the tsunami wavefront. A novel and noteworthy feature is that they also maximize in the backward direction of propagation west of the epicenter including the region where tsunami did not travel. This is owing to the large amplitude of the tsunami and associated large horizontal inhomogeneity westward of epicenter associated to coastal tsunami height amplification which lead to the excitation of strong AGWs in the atmosphere and so CADs/CIDs.

3. The high frequency ( $\sim 10$ minutes) component of the simulated CADs/CIDs and magnetic anomalies in the $F$ region develops within 6-7 minutes after the initiation of the tsunami, suggesting the importance of monitoring the high-frequency atmospheric/ionospheric anomalies for the early warning, as reported on observations.

\section{References}

Artru, J., V. Ducic, H. Kanamori, P. Lognonne, Murakami (2005), lonospheric detection of gravity waves induced by tsunamis, Geophys. J. Int., 160, 840a doi:10.1111/j.1365246X.2005.02552.x.

Calais, E., and J. B. Minster (1995), GPS detection of ionospheric perturbations following the January 17, 1994, Northridge earthquake, Geophys. Res. Lett., 22, 1045a 1048, doi:10.1029/95GL00168.[AGU]

Heki, K., and J. Ping (2005), Directivity and apparent velocity of the coseismic ionospheric disturbances observed with a dense GPS array, Earth Planet. Sci. Lett., 236, 845-855, doi:10.1016/j.epsl.2005.06.010.[CrossRef]

Hebert,H., A. Sladen, and F. Schindel (2006), Numerical modeling of the great 2004 Indian Ocean tsunami: Focus on the Mascarene Islands, Bulletin of the Seismological Society of America; January 2007; v. 97; no. 1A; p. S208S222.

Kherani, E.A., P. Lognonne, N. Kamath, F. Crespon and R. Garcia, Response of the ionosphere to the seismic triggered acoustic waves: electron density and electromagnetic fluctuations, 1-13, 176, Geophys J. Int., doi : 10.1111/j.1365-246X.2008.03818.x, 2009.

Lognonné, P., C. Clévédé and H. Kanamori, Normal mode summation of seismograms and barograms in an spherical Earth with realistic atmosphere, Geophys. J. Int., 135, 388-406, doi : 10.1046/j.1365-246X.1998.00665.x 
,1998.

Makela, J. J., P.Lognonné, H.Hébert, T. Gehrels, L. Rolland, S. Allgeyer, E.A. Kherani, G.Occhipinti, E.Astafyeva, P.Coisson, A.Loevenbruck, E.Clévédé, M.C. Kelley, J.Lamouroux (2011), Imaging and modeling the ionospheric airglow response over Hawaii to the tsunami generated by the Tohoku earthquake of 11 March 2011, Geophys. Res. Lett., 38, L00G02, doi:10.1029/2011GL047860.

Occhipinti, G., E.A. Kherani, P. Lognonné (2008), Geomagnetic dependence of ionospheric disturbances induced by tsunamigenic internal gravity waves, Geophys. J. Int, 753-755, 173, doi: 10.1111/j.1365246X.2008.03760.x.

Rolland, L.M., P. Lognonné, E.Astafyeva, E. A. Kherani, N. Kobayashi, M. Mann and H. Munekane (2011b), The resonant response of the ionosphere imaged after the 2011 Tohoku-Oki earthquake, Earth Planets Space, doi:10.5047/eps.2011.06.020, 2011.

\section{Acknowledgments}

EAK thanks FAPESP for the grant under process number 2007/00104-0 and INPE for providing research facility.
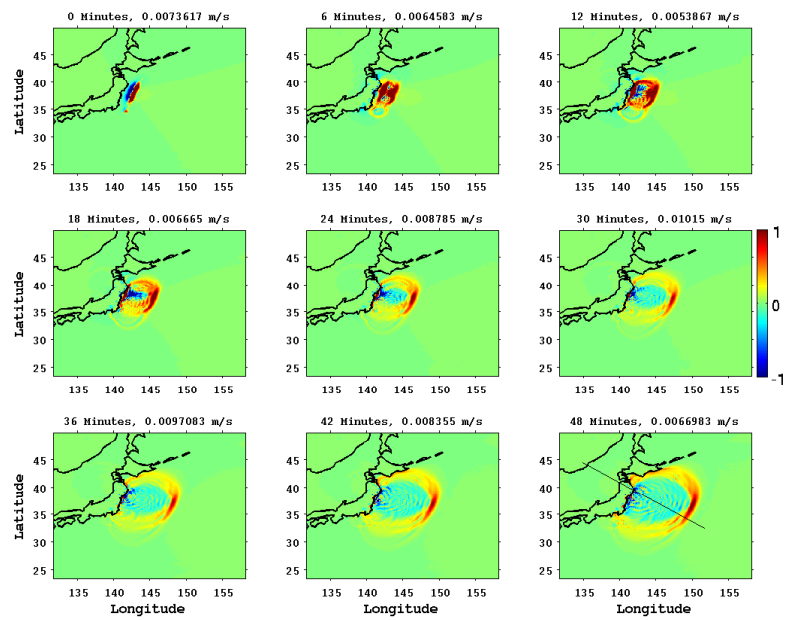

Figure 1: The tsunami wave field WT (normalized to the instantaneous maximum value) at few selected time after the tsunami origin time 5:46:23 UT. At the top of each panel, the time after the origin time and the maximum value of WT is shown. The line drawn in last panel represents the horizontal cut along which Travel-time diagrams in Figure 9 are plotted.
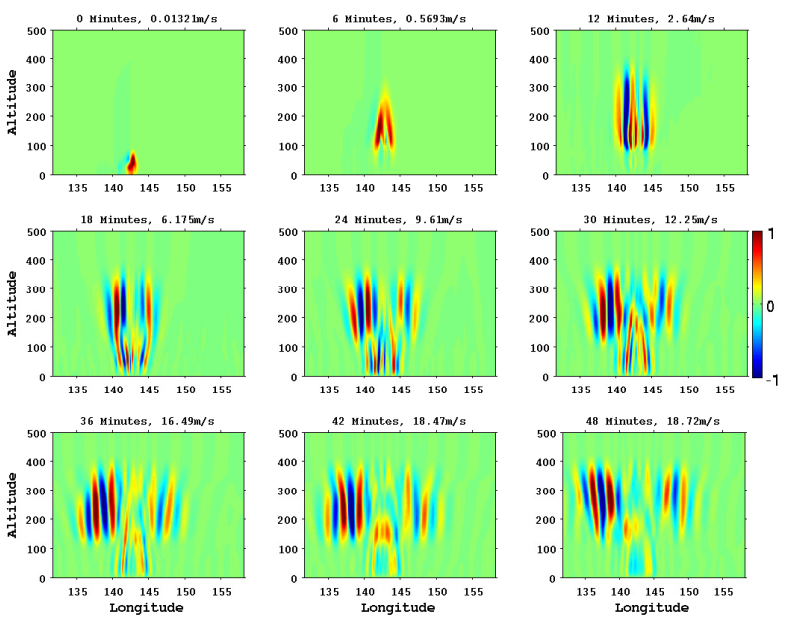

Figure 2: Vertical propagation of the simulated CADs (referred to the wind Wp component of AGWs, where Wp represents the component of the wind outward normal to the Earth's magnetic field.): Wp (normalized to the instantaneous maximum value) at altitude-longitude plane at fixed latitude $=38.32^{\circ}$ is plotted during few selected time after the tsunami origin time 5:46:23 UT. At the top of each panel, the time after the origin time and maximum value of wind amplitude is shown.
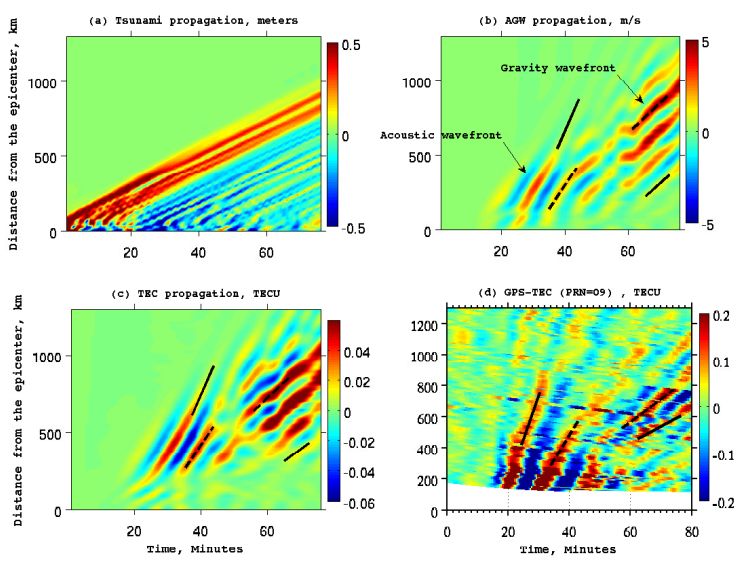

Figure 3: Travel-time diagram: The time vs epicenter distance variation (along the cut as shown in the Figure 1) of the (a) Input tsunami wave-field, (b) simulated CADs, (c) simulated CIDs (d) observed TEC or CIDs from GPS $(P R N=09)$. The solid lines in (b-c) represent the reference lines with slopes corresponding to the upper and lower limits $800 \mathrm{~m} / \mathrm{s}, 250 \mathrm{~m} / \mathrm{s}$ respectively. The dashed lines in $(b-c)$ represent the intermediate velocities equals to the $600 \mathrm{~m} / \mathrm{s}$ and $300 \mathrm{~m} / \mathrm{s}$ respectively. In (d), clockwise, four lines represent the velocities equals to the $1 \mathrm{~km} / \mathrm{s}, 550 \mathrm{~m} / \mathrm{s}$, $270 \mathrm{~m} / \mathrm{s}$ and $170 \mathrm{~m} / \mathrm{s}$ respectively. 